\title{
Factors influencing SARS-CoV-2 infection rate in Belgian nursing home residents during the first wave of COVID-19 pandemic
}

\section{Original Paper}

Cite this article: Peckeu-Abboud L, van Kleef E, Smekens T, Latour K, Dequeker S, Int Panis L, Laga M (2022). Factors influencing SARSCoV-2 infection rate in Belgian nursing home residents during the first wave of COVID-19 pandemic. Epidemiology and Infection 150, e72, 1-7. https://doi.org/10.1017/ S0950268822000334

Received: 13 September 2021

Revised: 10 February 2022

Accepted: 14 February 2022

Keywords:

Belgian nursing homes; risk factors analysis; SARS-CoV infection rate

Author for correspondence:

L. Peckeu-Abboud, E-mail: Ipeckeu@itg.be
L. Peckeu-Abboud ${ }^{1}$ (D), E. van Kleef ${ }^{1}$ (D), T. Smekens ${ }^{1}$, K. Latour ${ }^{2}$, S. Dequeker ${ }^{2}$, L. Int Panis ${ }^{2}$ and M. Laga ${ }^{1}$

${ }^{1}$ Institute of Tropical Medicine (ITM), Antwerp, Belgium and ${ }^{2}$ Sciensano, Brussels, Belgium

\begin{abstract}
In April 2020, Belgium experienced high numbers of fatal COVID-19 cases among nursing home $(\mathrm{NH})$ residents. In response, a mass testing campaign was organised testing all NH residents and staff. We analysed the data of Flemish NHs to identify institutional factors associated with increased SARS-CoV-2 infection rates among $\mathrm{NH}$ residents. Cross-sectional study was conducted between 8 April and 15 May 2020. Data collected included demographics, group category (i.e. staff or resident), symptom status and test result. We retrieved additional data: number of beds and staff, type of beds (level of dependency of residents) and ownership (public, private for profit/non-profit institutions). Risk factor analysis was performed using negative binomial regression. In total, 695 NHs were included, 282 (41\%) had at least one resident tested positive. Higher infection rate among residents was associated with a higher fraction of RVT beds, generally occupied by more dependent residents (incidence rate ratio (IRR) $1.97 ; 95 \%$ CI $1.00-3.86$ ) and higher staff infection rate (IRR 1.89; 95\% CI 1.68-2.12). No relationship was found between other investigated NH characteristics and infection rate among residents. Staff-resident interactions are key in SARS-CoV-2 transmission dynamics. Vaccination, regular staff testing, assessment of infection prevention and control strategies in all NHs are needed to face future SARS-CoV-2 epidemics in these settings.
\end{abstract}

\section{Introduction}

High rates of morbidity and case fatality related to severe acute respiratory syndrome coronavirus 2 (SARS-CoV-2) outbreaks in nursing homes (NHs) for the elderly have been reported across Europe [1]. In Belgium, the first cases of COVID-19 in the general population were notified from February 2020. By May 2020, Belgium experienced one of the highest numbers of reported fatal COVID-19 cases globally. However, during these first months of the pandemic, all deaths reported among possible COVID-19 cases qualified as COVID-19-related deaths [2]. This broad case-definition partially contributed to the relatively high reported COVID-related death burden in Belgium at that time. Importantly, in these early phases, NHs accounted for more than half (413 deaths/million inhabitants, 51\%) of the possible cases reported [1].

In response, Belgium implemented a mass testing campaign among all Belgian NHs in early April. The campaign intended to test all NH residents and staff, hence provide situational awareness and guidance for the national COVID-19 response. These cross-sectional data revealed a $2 \%$ and $4 \%$ SARS-CoV-2 positivity rate, respectively, among $\mathrm{NH}$ staff and residents tested $(N=321$ and 553) [3].

In addition to the extent, these nation-wide testing efforts highlighted the heterogeneity of SARS-CoV-2 spread among Belgian NHs. Over half of the NHs reported no infections among residents or staff, while others experienced large outbreaks with more than 10 residents infected [4]. To date, institutional characteristics associated with higher infection rates among Belgian NH residents during the early pandemic phase remain unclear. One of the few larger scale studies conducted in Europe, i.e. England, identified reduced transmission rates related to factors such as staff to bed ratio, size of the $\mathrm{NH}$ and age of residents [5]. In contrast, large-scale regional studies from the United States and Scotland found no such associations $[6,7]$. Furthermore, provision and organisational infrastructure of long-term elderly care vary between countries and regions, making the extrapolation of existing findings to other geographical regions not self-evident.

In this study, we conducted a NH-level analysis on institutional risk factors associated with the proportion of $\mathrm{NH}$ residents tested positive for SARS-CoV-2 in the largest Belgian region of Flanders. Hence, we aimed to identify factors contributing to SARS-CoV-2 positivity rate in long-term elderly care settings, in the early phase of the pandemic. 


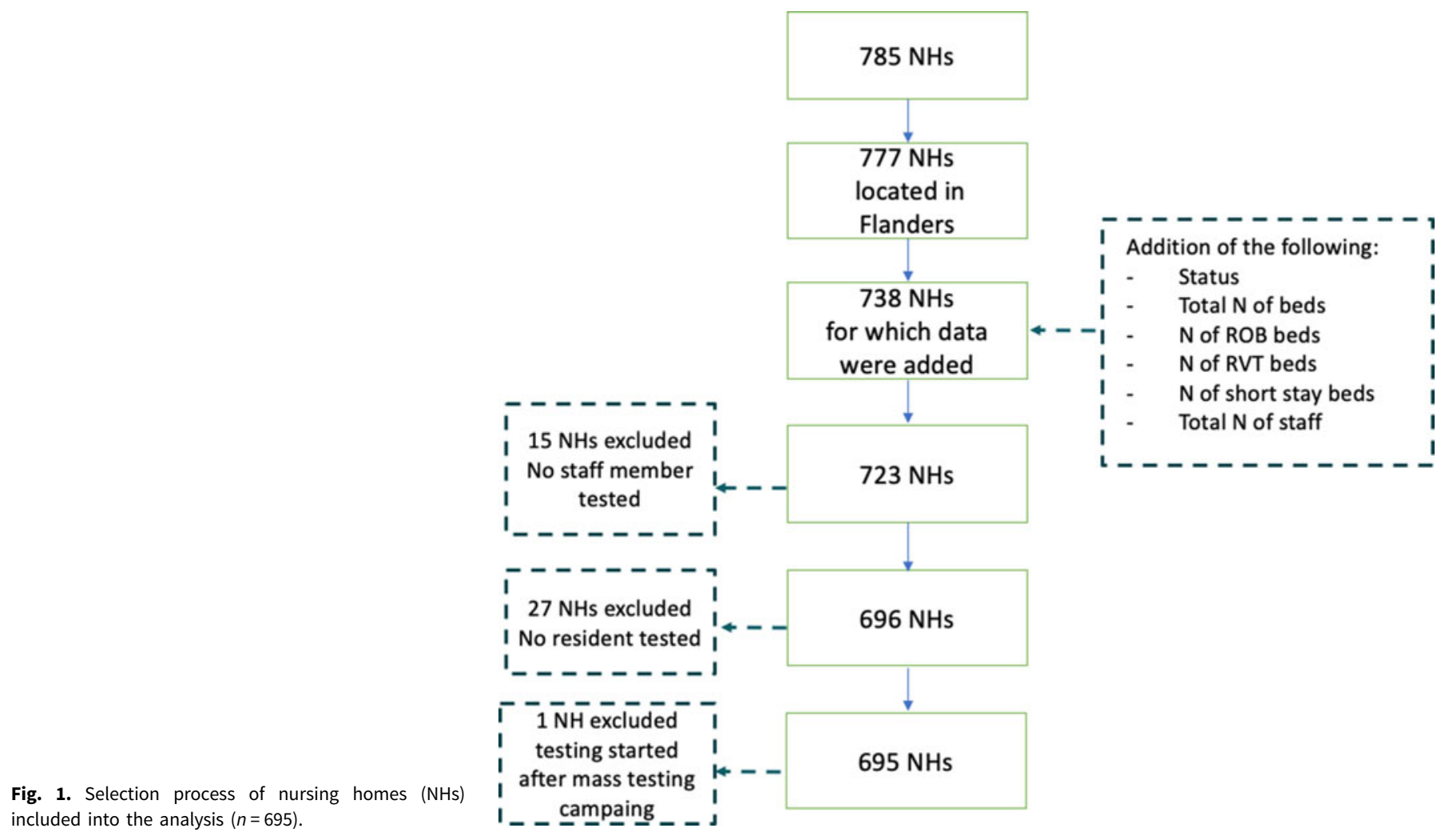

\section{Methods}

\section{National survey among nursing homes}

The COVID-19 testing campaign was implemented between 8 April and 15 May 2020. All three Belgian regions organised an individual campaign for their region. All data were centralised in a national database curated by the Belgian national public health institute Sciensano (https://www.sciensano.be). A detailed description of the national survey's study design has been described elsewhere [8]. In brief, each NH organised staff and resident testing on a single day or over the course of several days (depending on their logistics and capacity available). Nasopharyngeal swabs were collected by occupational physicians of the NHs and real-time-PCR testing was performed. Collected data were available to a medical doctor on a secured platform. All participants were informed about the testing campaign and orally agreed to participate. Here, we focus on data collected from NHs located in the Flanders region, including an additional eight NHs located in the Brussels region that fall under Flanders authority. In Flanders, for logistic reasons (such as test availability), priority in testing was given to facilities $(n=55)$ reporting either the highest percentage of residents with COVID-19-related symptoms, the highest percentage of symptomatic staff, or a combination of both. To still allow for rapid insight in the general COVID-19 situation among Flemish NHs, an additional randomly selected number of NHs $(n=30)$ reporting low or no COVID-19 cases among staff or residents (at the time of selection) were also included in the early April testing phase (815 April 2020) $[9,10]$. Following this early testing phase, a coordinated and harmonised test strategy for the remaining NHs of the entire region was defined. Data collected included test ID number, age, gender, postal code, group category (i.e. staff or resident), $\mathrm{NH}$ name, symptom status at the time of testing and test result [8]. Additional data were made available by Sciensano, and linked to the mass testing data restricted to the Flemish region. These data included NH size (number of beds and number of NH staff members), type of beds available (number of beds for highly caredependent residents (rust-en verzorgingstehuis, abbreviated to RVT beds), number of beds suitable for non-care-dependent residents (rustoord voor bejaarden, abbreviated to ROB beds) and number of short stay beds) and NH ownership (public institutions, private non-profit institutions and private for-profit institutions).

\section{Selection of $\mathrm{NH}$ included in this analysis}

Among all 1542 NHs registered in Belgium, 814 are located in Flanders, 147 in Brussels region (including eight NHs that fall under Flanders' authority), 573 in Wallonia (including eight that fall under German-speaking Community's authority). Survey methods differed between the three regions, and we analysed data of the largest, i.e. Flemish region, which was made available to us in consultation with the Flemish Public Health Authority. In total, we received data from 785 Flemish NHs, of which 695 were included in our analysis. We excluded NHs for which no staff members or residents were tested, or NHs where testing was conducted outside the scope of the mass testing campaign (Fig. 1).

\section{Data preparation}

We aimed to identify NHs characteristics associated with SARS-COV-2 test positivity rate among residents. To allow for such $\mathrm{NH}$-level analyses, an aggregated database was created. We computed indicators based on the available data and where needed, used guidance from literature (Table 1).

Test positivity rate (also called infection rate, is the proportion of staff members and residents that tested positive for 
SARS-CoV-2) was calculated by dividing the number of residents or $\mathrm{NH}$ staff members testing positive by the total number of residents or NH staff members tested during the mass testing campaign per $\mathrm{NH}$, respectively. The proportion of asymptomatic cases (staff or residents) was defined as the number of residents or NH staff members testing positive but presenting no symptoms of an infection divided by the number of residents or $\mathrm{NH}$ staff members testing positive (with or without symptoms). The number of total $\mathrm{NH}$ beds was divided by 20 and fitted as a continuous variable, similar to [6], allowing for IRRs per 20-bed increase, which, from a clinical perspective, was considered more meaningful. The proportion of RVT beds was defined as the number of RVT beds (beds for highly care-dependent residents) divided by the total number of beds included in the NH. Since the most affected NHs were given priority in the mass testing campaign, we accounted for individual $\mathrm{NH}$ testing starting dates in the analyses (i.e. time as a continuous variable). The size of the outbreak was defined according to the Belgian national definition for institutional outbreak size: small outbreak ( 1 or 2 SARS-CoV-2 cases reported), medium outbreak (3-9 SARS-CoV-2 cases reported) and large ( $\geqslant 10$ SARS-CoV-2 cases reported).

\section{Statistical analyses}

Descriptive analysis on the NHs characteristics was performed, mean and standard deviation (S.D.) were estimated for normally distributed continuous variables; median and interquartile ranges (IQR) were estimated for non-normally distributed variables. Frequencies were described for categorical variables.

We first explored the association between staff and resident positivity rates (infection rates) as a proxy measure for staff and resident interactions by assessing the non-parametric Spearman's correlation. To further identify risk factors for $\mathrm{NH}$-level test positivity rates, we fitted a negative binomial regression model with an off-set term (i.e. the total number of residents per NHs) to the per-NH proportions of residents tested positive. To measure the relative change (in percentage) between positivity rate among residents and staff members, the staff members' positivity rate was log-transformed. Univariate and multivariate incidence rate ratios (IRRs) and their 95\% confidence intervals (CIs) were estimated. Univariate analyses were performed on computed $\mathrm{NH}$ characteristics. $\mathrm{NH}$ characteristics with a $P$-value $\leqslant 0.25$ were included in the multivariate analysis. In the multivariate analysis, a $P$-value $\leqslant 0.05$ was considered significant.

All analyses were performed using $\mathrm{R}$ version 3.6.3, using tidyverse and ggplot packages for our exploratory analyses, the MASS package was used for regression model fitting. Scripts are accessible via the GitHub repository: https:/github.com/Laureneitm/ Belgian_NH_analysis

The study was approved by the Institutional Review Board of the Institute of Tropical Medicine.

\section{Results}

\section{Characteristics of the NHs and their population}

A total of 66209 (84\%) residents and 62989 (93\%) NH staff members were tested during the testing campaign among 695 NHs included in our study. Nearly half of these NHs $(n=338$, $49 \%$ ) were large NHs ( $>100$ beds), with the majority of beds (56\%) comprising of 'RVT beds'. NHs were largely private nonprofit institutions $(n=385,55 \%)$ (Table 1$)$.
Table 1. Characteristics of Flemish nursing homes (NHs) $(n=695)$

\begin{tabular}{|c|c|}
\hline NH characteristics & All NHs $(n=695)$ \\
\hline \multicolumn{2}{|l|}{ Number of beds, $n(\%)$} \\
\hline$\leqslant 20$ beds & $5(<1)$ \\
\hline $21-40$ beds & $35(5)$ \\
\hline $41-60$ beds & $63(9)$ \\
\hline $61-80$ beds & $119(17)$ \\
\hline $81-100$ beds & $135(19)$ \\
\hline$>100$ beds & $338(49)$ \\
\hline \multicolumn{2}{|l|}{ Ownership, $n(\%)$} \\
\hline Public institution & $179(26)$ \\
\hline Private non-profit institution & $385(55)$ \\
\hline Private for profit institution & $131(19)$ \\
\hline \multicolumn{2}{|l|}{ Type of beds, $n(\%)$} \\
\hline Short stay beds & $2207(3)$ \\
\hline ROB beds ${ }^{a}$ & $31095(41)$ \\
\hline RVT beds ${ }^{b}$ & $41912(56)$ \\
\hline NH with no infection, $n(\%)$ & $324(47)$ \\
\hline NH with 1 or 2 cases, $n(\%)$ & $155(22)$ \\
\hline NH with 3-9 cases, $n(\%)$ & $109(17)$ \\
\hline $\mathrm{NH}$ with $\geq 10$ cases, $n(\%)$ & $107(15)$ \\
\hline Ratio NH staff members/residents & $0.92(0.74-1.11)$ \\
\hline \multicolumn{2}{|l|}{ Residents } \\
\hline$n(\%)$ & $78589(54)$ \\
\hline$n$ tested $(\%)$ & $66209(51)$ \\
\hline$n$ tested per $\mathrm{NH}$, median (IQR) & $89(62-117)$ \\
\hline Age (in years), median (IQR) & $86(85-87)$ \\
\hline$n$ tested positive (\%) & $2492(4)$ \\
\hline $\begin{array}{l}n \text { asymptomatic and tested positive } / n \text { tested } \\
\text { positive (\%) }\end{array}$ & $1896 / 2492(76)$ \\
\hline \multicolumn{2}{|l|}{ NH staff members } \\
\hline$n(\%)$ & $67383(46)$ \\
\hline$n$ tested $(\%)$ & $62989(49)$ \\
\hline$n$ tested per $\mathrm{NH}$, median (IQR) & $82(57-116)$ \\
\hline Age (in years), median (IQR) & $42(40-44)$ \\
\hline$n$ tested positive (\%) & $1430(2)$ \\
\hline $\begin{array}{l}n \text { asymptomatic and tested positive/ } n \\
\text { tested positive }(\%)\end{array}$ & $1035 / 1430(72)$ \\
\hline
\end{tabular}

${ }^{\mathrm{a}}$ Beds for non-care-dependent residents.

${ }^{\mathrm{b}}$ Beds for highly care-dependent residents.

During the mass testing campaign, 324 (47\%) of NHs did not report a single SARS-CoV-2 infection among residents. Among the NHs that reported positive cases, the majority $(n=155$, $22 \%$ ) reported one or two SARS-CoV-2 cases (a small outbreak according to the Belgian national definition for institutional outbreak size). This was followed by $17 \%(n=109)$ and $15 \%$ $(n=107)$ of NHs reporting medium (3-9 cases) and large $(\geqslant 10$ cases) outbreaks, respectively. Per $\mathrm{NH}$, a median of 89 residents 


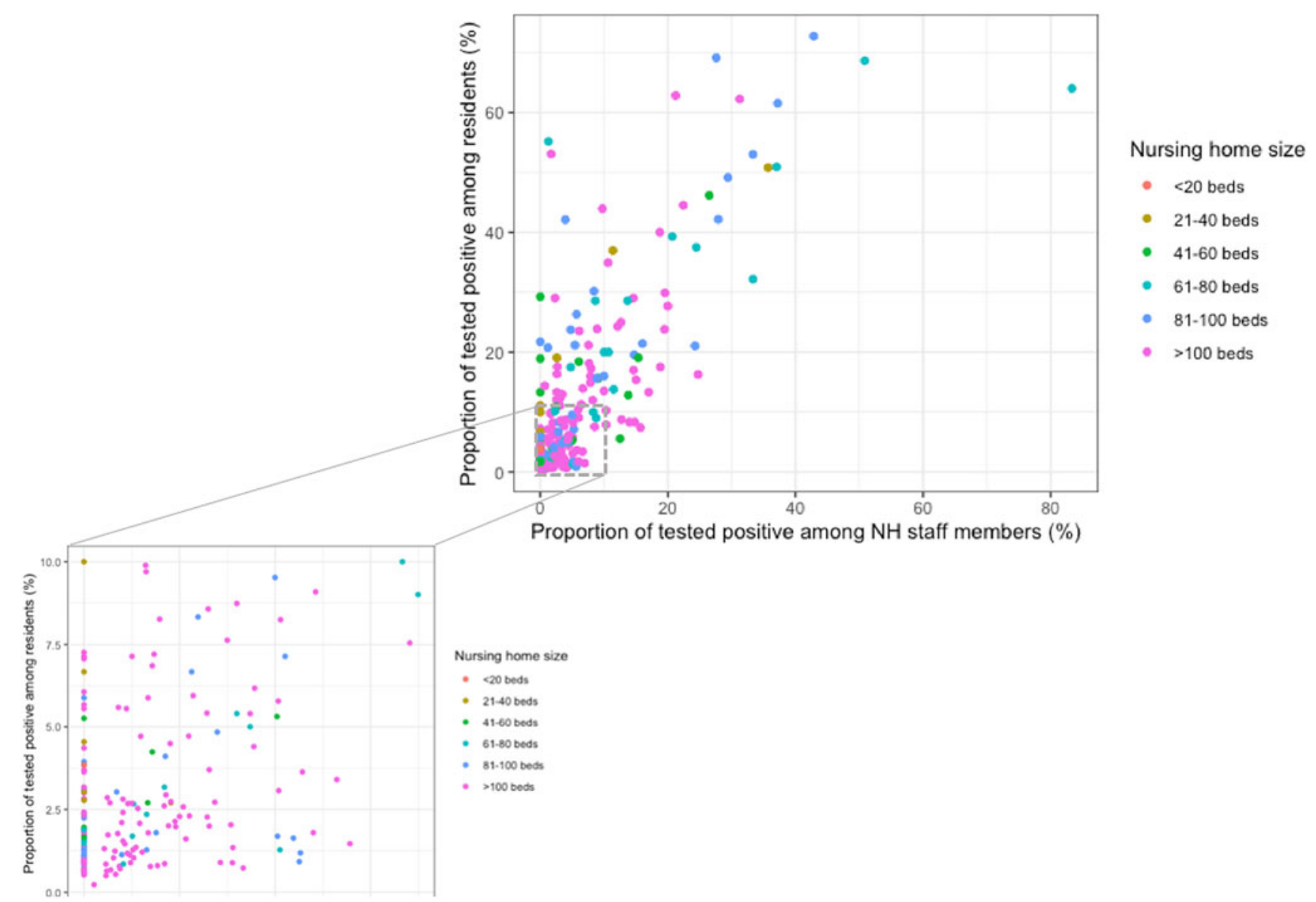

Fig. 2. Proportion of tested positive among residents and NH staff members in the 282 Flemish nursing homes (NHs) with a proportion of tested positive residents above 0 (Spearman's correlation coefficient $=0.63, P$-value $<0.001$ ).

(IQR 62-117) and 82 (IQR 57-116) NH staff members were tested on average. Among $\mathrm{NH}$ staff members, the median age was 42 years (IQR 40-44), compared to 86 years (IQR 85-87) among residents. Among the residents and $\mathrm{NH}$ staff members that tested positive, high percentages of asymptomatic cases were reported $(76 \%$ and $72 \%$, respectively, Table 1$)$.

\section{SARS-CoV-2 infections among NH staff and residents by institution}

For the $282(41 \%)$ institutions with $\geqslant 1$ infected residents reported, the infection rates varied from $0.2 \%$ to $72 \%$ (median of $4 \%$ ) among residents and from $0 \%$ to $83 \%$ among $\mathrm{NH}$ staff members (median of 2\%) (Fig. 2). We found a significant positive correlation between the proportion of staff members and residents tested positive, respectively (Spearman's correlation coefficient $=$ $0.63, P$-value $<0.001)$. The majority of NHs with $\geqslant 1$ cases concerned large NHs $(n=174,62 \%)$. Nonetheless, on visual inspection, reported infection rates among staff and/or residents did not appear to relate to NHs size, which was further explored in the multi-variate analysis (Fig. 2).

\section{Risk factor analyses on the proportion of tested positive residents}

We considered nine putative $\mathrm{NH}$ characteristics for increased SARS-CoV-2 positivity among $\mathrm{NH}$ residents at the $\mathrm{NH}$ level
(Table 2). Following our univariate analyses, we included the proportion of RVT beds (a proxy for the degree of resident dependency), resident and staff age, and the proportions of staff tested positive and asymptomatic cases among those tested positive (for both residents and $\mathrm{NH}$ staff) in a multivariate regression model.

The multivariate analysis (model fitted on $196 \mathrm{NHs)} \mathrm{revealed} \mathrm{a}$ positive association between the proportion of residents and staff tested positive (IRR 1.89; 95\% CI 1.68-2.12 for each per cent increase). Importantly, a one unit increase in the proportion of RVT beds was associated with a twofold increase in the proportion of residents tested positive (IRR 1.97; IQR 1.00-3.86). No significant association was found for the other $\mathrm{NH}$ characteristics.

\section{Discussion}

In this study, we explored $\mathrm{NH}$ characteristics that may have predisposed NHs in Flanders to SARS-CoV-2 outbreaks during Belgium's first wave of the COVID-19 epidemic. The mass testing campaign revealed that over half $(53 \%)$ of Flemish NHs had at least one SARS-CoV-2 infection identified among $\mathrm{NH}$ residents and staff, with $15 \%$ of NHs facing large outbreaks ( $\geqslant 10$ cases). The risk of infection among $\mathrm{NH}$ residents was significantly increased with an increased infection rate among $\mathrm{NH}$ staff members and a higher fraction of RVT beds. No associations were found between SARS-CoV-2 positivity rates and size of the $\mathrm{NH}$ expressed in number of beds, type of $\mathrm{NH}$ (public or private), 
Table 2. Incidence rate ratio (IRR) (univariate and multivariate negative binomial regression) of associations between the proportion of tested positive among residents and nursing home $(\mathrm{NH})$ characteristics

\begin{tabular}{|c|c|c|c|c|}
\hline \multirow[b]{2}{*}{ NH characteristics } & \multicolumn{2}{|c|}{ Univariate analysis } & \multicolumn{2}{|c|}{ Multivariate analysis ${ }^{a}$} \\
\hline & IRR $(95 \% \mathrm{Cl})$ & $P$-value & $\operatorname{IRR}(95 \% \mathrm{Cl})$ & $P$-value \\
\hline$n$ total beds (=total population of resident) (per 20 beds) ( 0 missing value) & $1.01(0.95-1.08)$ & 0.86 & - & - \\
\hline Proportion of RVT beds ${ }^{b}$ ( $n$ RVT beds/ $n$ total beds) ( 0 missing value) & $2.95(1.16-7.03)$ & 0.02 & $1.97(1.00-3.86)$ & 0.05 \\
\hline Ratio NH staff members/residents (0 missing value) & $1.28(0.75-2.29)$ & 0.37 & - & - \\
\hline Mean age NH staff members (in years) ( 0 missing value) & $1.06(1.02-1.12)$ & 0.02 & $1.02(1.00-1.05)$ & 0.26 \\
\hline Mean age residents (in years) (0 missing value) & $0.97(0.90-1.02)$ & 0.12 & $1.03(1.00-1.08)$ & 0.07 \\
\hline Test positivity rate among $\mathrm{NH}$ staff members ( 0 missing value) & $1.97(1.85-2.10)$ & $<0.001$ & $1.89(1.68-2.12)$ & $<0.001$ \\
\hline $\begin{array}{l}\text { Proportion of asymptomatic NH staff members ( } n \text { asymptomatic + tested positive } \mathrm{NH} \\
\text { staff members } / n \text { positive } \mathrm{NH} \text { staff members) ( } 0 \text { missing values) }\end{array}$ & $0.40(0.19-0.82)$ & 0.001 & $0.85(0.58-1.25)$ & 0.41 \\
\hline $\begin{array}{l}\text { Proportion of asymptomatic residents ( } n \text { asymptomatic }+ \text { tested positive residents } / n \\
\text { positive residents) ( } 0 \text { missing values) }\end{array}$ & $0.19(0.10-0.35)$ & $<0.001$ & $1.05(0.63-1.72)$ & 0.82 \\
\hline Ownership (0 missing value) & $1.06(0.80-1.41)$ & 0.68 & - & - \\
\hline
\end{tabular}

${ }^{\mathrm{a}}$ Degrees of freedom $=195$.

beds for highly care-dependent residents.

mean age of the residents and staff, or proportion of asymptomatic tested positive cases among residents and staff.

Both the associations of higher staff infection rate as well as a higher ratio of RVT beds likely point to a key role of interactions between staff members and residents in SARS-CoV-2 transmission dynamics in $\mathrm{NH}$ settings. For the latter, RVT beds or rooms are generally occupied by residents requiring higher levels of care, and therefore requiring more staff contact, whilst contact with other residents is limited. Available stock of personal protective equipment (PPE) as well as training on their proper use where limited in the early phase of the pandemic in Belgium. Frontline reports revealed that the lack of equipment and clear infection prevention and control (IPC) strategies (especially testing and staff cohorting) resulted in increased risk of onward SARS-CoV-2 transmission among staff and residents [11]. Along with these results, Telford et al., investigated among a sample of $23 \mathrm{NHs}$ in Georgia 33 key indicators from five IPC categories (hand hygiene, disinfection, social distancing, PPE, and screening for symptoms and elevated temperature) and found that differences between higher- and lower-prevalence NHs occurred most frequently in the social distancing and PPE categories [12]. In our study, we did not have access to reliable data on the availability and use of PPE, nor IPC strategies implemented. Nonetheless, lack of PPE supply and suboptimal screening and testing strategies were universal across Belgian NHs [13].

We found that $47 \%$ of the NHs in Flanders did not have any SARS-CoV-2 infection detected in the early April-May 2020 period, implying a marked fraction of $\mathrm{NH}$ staff members and residents remained vulnerable to SARS-CoV-2 infections. Similar observations were made in regional studies from North America and Europe, where a large proportion of NHs were still susceptible to SARS-COV-2 infections after May 2020 [6, 14-16]. Post Belgium's testing campaign, several initiatives were taken in Flanders to overcome the COVID-19 crisis in NHs. Strategies to exchange personnel between different settings including hospitals and NHs were implemented [17] and support was provided on medical expertise, training, material and medication. As well as organisation of material supply and deliveries such as PPE within
Flemish NHs [18]. Despite these investments, during the autumn second wave $2020,73 \%$ and $49 \%$ of Belgian NHs reported $\geqslant 2$ and $\geqslant 10$ confirmed cases, respectively [19] and at the moment of writing, $96 \%$ of Belgian NHs reported $\geqslant 2$ possible or confirmed cases [20]. SARS-CoV-2 transmission dynamics and IPC in general in NHs come with a multifactorial set of challenges different from more controllable clinical settings, such as adherence and understanding of IPC guidelines from the residents themselves [21].

In addition, mitigating staff-resident transmission risk in the early phase of the pandemic, as well as over the course of the pandemic, has been challenged by the recognised role of asymptomatic and pre-symptomatic carriers in the SARS-CoV-2 transmission [22-24]. In an earlier analysis of the (national) mass testing campaign data, Hoxha et al. concluded that high levels of identified a- and pre-symptomatic carriers (means of $74 \%$ and $75 \%$ of infected staff and residents, respectively) point towards nonsymptomatic individuals having comprised an important driver of transmission within Belgian NHs. Our analyses did not reveal an increased $\mathrm{NH}-$ level resident infection rate related to the proportion of asymptomatic infections among staff or residents. However, this may be a result of the limited variation in asymptomatic carriage across all participating $\mathrm{NHs}$ (median $=100 \%$ (IQR 59-100) and $100 \%(71-100)$ for staff and residents, respectively). Moreover, due to the cross-sectional nature of our study, onward transmission could not be established, nor were CT-values available to determine what proportion of asymptomatic carriers was likely 'infectious'. Longitudinal studies and seroprevalence surveys on staff and residents could enhance our understanding of SARS-CoV-2 transmission in NHs.

While the above underlines the critical importance of good IPC practices; the availability of effective vaccines against COVID-19 has made active immunisation of staff and residents an essential part of infection prevention strategies in long-term care. In Belgium, vaccination campaigns started in December 2020. All residents and staff of the Belgian NHs were offered vaccination. By 24 March (end of the vaccination campaign in NHs), the vaccine coverage among staff varied by region, with $86 \%$ in Flanders, 58\% in Wallonia and $47 \%$ in Brussels [25]. The relative 
low acceptance among healthcare staff working with the most vulnerable population has initiated debates on compulsory vaccination for healthcare professionals. Our work reinforces the idea that maximum protection (by reaching and sustaining high immunisation coverage rates) and prevention through vaccination among staff of $\mathrm{NHs}$ is essential. Compulsory vaccination may be effective in preventing disease outbreaks, but it may also lead to negative vaccination attitudes decreasing vaccination uptake [26, 27]. Governments should take such factors in consideration when implementing vaccination policies.

We found no association between the number of beds (with potentially higher number of residents exposed), and the infection rate among residents. This is in line with two studies from Scotland and Canada involving, respectively, 189 care homes and $618 \mathrm{NHs,}$ where no relationship between care home number of beds and SARS-CoV-2 incidence was detected [6, 28]. Moreover, Abrams et al. found that larger facility size was significantly related to the probability of having a COVID-19 case (OR 6.52, $P<0.001$, for large facilities $v s$. small ones) [7]. In contrast, Shallcross et al. found higher occupancy to affect SARS-CoV-2 infection rates in NHs settings in England [29]. Therefore, evidence on the role of $\mathrm{NH}$ size in transmission dynamics of SARS-CoV-2 remains unclear and potential confounders (e.g. number of beds per room) could be more informative on the relationship between $\mathrm{NH}$ size and SARS-CoV-2 epidemics.

It is well known that older ages result in immune senescence, making elderly care residents vulnerable to emerging infectious diseases such as SARS-CoV-2 [30]. Shallcross et al. reported a significant association between increasing age and the odds of infection in residents (aOR 1.01; 95\% CI 1.01-1.03) [5]. Belgium's elderly care resident population is relatively old, and average length of NHs stay is about 2 years [31]. The limited age range in Belgian NHs may be a potential explanation for age not constituting a risk factor to SARS-CoV-2 infection rates in our study.

Our study has several strengths. We are among the few studies that used large-scale survey data including $695 \mathrm{NHs}$, representing $85 \%$ of all the NHs located in Flanders $(n=814)$. Additionally, we linked the mass testing data to routinely collected data hence providing relevant insights on the role of $\mathrm{NH}$ characteristics. While the survey was implemented with the aim to guide the national response strategy and its design, some limitations have to be recognised. For facilities with more staff and residents than the number of tests assigned, the prioritisation was left at the discretion of the structure. Therefore, it is possible that previously identified infections among residents (before the mass testing campaign) were not tested at the time of the mass testing campaign and were therefore not included in our analysis. This could have led to an underestimated proportion of residents tested positive in some NHs. Furthermore, known cases of staff members in quarantine, not present at the $\mathrm{NH}$ during the days of the mass testing campaign were not tested. Therefore, infection rate among staff could be underestimated as well. The number of variables that could be explored was limited to the variables collected during this mass testing campaign. Variables such as isolation procedures for residents, staff cohorting and the number of staff working at different sites could have been potential factors influencing the proportion of positive tests. In addition to the quantitative study, qualitative data could have improved our understanding of the situation that each $\mathrm{NH}$ faced, giving more detailed information on limitations in the response capacity.

In conclusion, our analysis showed that two factors (positivity rate among staff and fraction of RVT beds) both related to staff-resident interactions influenced the infection rate of SARS-CoV-2 among residents in the Flemish NH during the first wave of the pandemic. Reducing the impact of COVID-19 in NH settings goes along with an adequate protection of staff members preventing transmission from and to the residents. We recommend the implementation of targeted interventions such as vaccination, regular staff testing, assessment of IPC strategies in all NHs to be able to face future (SARS-CoV-2) epidemics in these settings.

Acknowledgements. We thank Agentschap Zorg en Gezondheid AZ\&G for their support. We thank Sciensano for making the administrative data on $\mathrm{NHs}$ available.

Author contributions. LPA and EvK conceptualised the design and analyses and wrote the draft manuscript; TS contributed to the data analysis and interpretation; KL, SD and LIP supported the interpretation of results and revised the manuscript; ML contributed to the interpretation and revised the manuscript.

\section{Conflict of interest. None.}

Data availability statement. Data are available on request from the corresponding author, subject to ITM and AZ\&G disclosure controls to prevent identification of individuals.

\section{References}

1. Danis K et al. (2020) High impact of COVID-19 in long-term care facilities, suggestion for monitoring in the EU/EEA, May 2020. Eurosurveillance 25, 2000956.

2. Renard F et al. (2021) Establishing an ad hoc COVID-19 mortality surveillance during the first epidemic wave in Belgium, 1 March to 21 June 2020. Eurosurveillance 26, 2001402.

3. Sciensano (2020) COVID-19 - Bulletin epidemiologique du 26 mai 2020. https://covid-19.sciensano.be/sites/default/files/Covid19/COVID-19_Daily \%20report_20200526\%20-\%20FR.pdf.

4. Sciensano (2020) Surveillance COVID-19 en maisons de repos et maisons de repos et de soins. Rapport de la semaine 26. https://covid-19.sciensano. be/sites/default/files/Covid19/COVID-19_Surveillance_MR_MRS.pdf.

5. Shallcross L et al. (2021) Factors associated with SARS-CoV-2 infection and outbreaks in long-term care facilities in England: a national crosssectional survey. The Lancet Healthy Longevity 2, e129-e142.

6. Burton JK et al. (2020) Evolution and effects of COVID-19 outbreaks in care homes: a population analysis in 189 care homes in one geographical region of the UK. The Lancet Healthy Longevity 1, e21-e31.

7. Abrams HR et al. (2020) Characteristics of US nursing homes with COVID-19 cases. Journal of the American Geriatrics Society 68, 1653-1656.

8. Hoxha A et al. (2020) Asymptomatic SARS-CoV-2 infection in Belgian long-term care facilities. The Lancet. Infectious Diseases 21, e67.

9. Department Welzijn Volksgezondheid and Gezin (2020) https://www departementwvg.be/sites/default/files/media/20200412_Taskforce_dringend_ wob.pdf.

10. Agentschap zorg and Gezondheid (2020) https://www.zorg-en-gezondheid.be/11243-testkits-voor-vlaamse-woonzorgcentra.

11. Médecin sans frontières (2020) https://www.msf.org/left-behind-coronavirus-belgium-care-homes-report.

12. Telford CT et al. (2020) Assessment of infection prevention and control protocols, procedures, and implementation in response to the COVID-19 pandemic in twenty-three long-term care facilities in Fulton County, Georgia. medRxiv. doi: 10.1101/2020.08.13.20174466

13. Gillain S, Belche JL and Moreau JF (2020) COVID-19 epidemic in the nursing homes in Belgium. Journal of Nursing Home Research 6, 40-42.

14. Li Y et al. (2020) COVID-19 infections and deaths among Connecticut nursing home residents: facility correlates. Journal of the American Geriatrics Society 68, 1899-1906.

15. Rolland Y et al. (2020) Guidance for the prevention of the COVID-19 epidemic in long-term care facilities: a short-term prospective study. The Journal of Nutrition, Health and Aging 24, 812-816. 
16. Fisman DN et al. (2020) Risk factors associated with mortality among residents with coronavirus disease 2019 (COVID-19) in long-term care facilities in Ontario, Canada. JAMA Network Open 3, e2015957.

17. Department Welzijn Volksgezondheid and Gezin (2020) https://www. departementwvg.be/taskforce.

18. Department Welzijn Volksgezondheid and Gezin (2020) https://www. departementwvg.be/sites/default/files/media/20200415_Taskforce_wob.pdf.

19. Sciensano (2020) https://covid-19.sciensano.be/sites/default/files/Covid19/ COVID-19_Surveillance_MR_MRS.pdf.

20. Dequeker S et al. (2021) Surveillance COVID-19 en maisons de repos et maisons de repos et de soins. Bruxelles, Belgique: Sciensano; 2021 38p. https://covid-19.sciensano.be/sites/default/files/Covid19/COVID-19_Weekly_ Pub_NH_FR_2021_06_30.pdf.

21. Micocci M et al. (2020) Understanding COVID-19 testing pathways in English care homes to identify the role of point-of-care testing: an interview-based process mapping study. medRxiv. doi: 10.1101/2020.11. 02.20224550

22. Wei WE et al. (2020) Presymptomatic transmission of SARS-CoV-2 Singapore, January 23-March 16, 2020. Morbidity and Mortality Weekly Report 69, 411.

23. Tong ZD et al. (2020) Potential presymptomatic transmission of SARS-CoV-2, Zhejiang Province, China, 2020. Emerging Infectious Diseases 26, 1052.

24. Huang L et al. (2020) Rapid asymptomatic transmission of COVID-19 during the incubation period demonstrating strong infectivity in a cluster of youngsters aged 16-23 years outside Wuhan and characteristics of young patients with COVID-19: a prospective contact-tracing study. Journal of Infection 80, e1-e13.

25. Sciensano (2020) Rapport thématique: Surveillance de la vaccination COVID-19 dans les maisons de repos et maisons de repos et de soins belges. https://covid-19.sciensano.be/sites/default/files/Covid19/COVID-19_ THEMATIC\%20REPORT_SURVEILLANCE\%20DE\%20LA\%20VACCINAT ION\%20DANS\%20LES\%20MAISONS\%20DE\%20REPOS\%20BELGES.pdf.

26. Salmonn DA et al. (2006) Compulsory vaccination and conscientious or philosophical exemptions: past, present, and future. The Lancet 367, 436-442.

27. Betsch C and Böhm R (2016) Detrimental effects of introducing partial compulsory vaccination: experimental evidence. The European Journal of Public Health 26, 378-381.

28. Brown KA et al. (2021) Association between nursing home crowding and COVID-19 infection and mortality in Ontario, Canada. JAMA Internal Medicine 181, 229-236.

29. Dutey-Magni PF et al. (2021) Covid-19 infection and attributable mortality in UK long term care facilities: cohort study using active surveillance and electronic records (March-June 2020). medRxiv, 2020-07. doi: 10.1101/2020.07.14.20152629

30. Nikolich-Zugich J et al. (2020) SARS-CoV-2 and COVID-19 in older adults: what we may expect regarding pathogenesis, immune responses, and outcomes. Geroscience 42, 505-514.

31. European Social Policy Network (ESPN) (2018) Thematic report on challenges in long-term care, Belgium. https://ec.europa.eu/. 